\section{In vitro antifungal susceptibili- ty of candidemia agents and detection of their biofilm production by two different methods}

\section{Yasemin Oz, Iman Qoraan, Egemen Gokbolat}

Department of Microbiology, Faculty of Medicine, Eskisehir Osmangazi University, Eskisehir, Turkey

\begin{abstract}
Candida bloodstream infections are a significant cause of morbidity and mortality in hospitalized patients. The most important contribution of biofilm is the higher antifungal resistance than planktonic cells. We aimed to investigate the biofilm formation rate and antifungal susceptibility characteristics of our bloodstream isolates, and evaluate two different biofilm detection methods. A total of 200 bloodstream Candida isolates were included. The biofilms were formed on 96-well microtiter plates and measured by spectrophotometric percent transmittance and 2,3-bis(2methoxy-4-nitro-5-sulfo-phenyl)-2H-tetrazolium- 5-carboxanilide colorimetric assay. In addition antifungal susceptibilities of these isolates were evaluated against caspofungin, anidulafungin and amphotericin B by reference method. Biofilm production rate was considerably high among our bloodstream isolates. The most important biofilm producer species was $C$. tropicalis; C. glabrata had the lowest biofilm production rate. The consistency rate between biofilm detection methods was $66 \%$. Remarkable antifungal resistance was not observed among our isolates in general. In conclusion, biofilm production in Candida species is an important virulence factor, and its rate is considerably high in bloodstream isolates. At present, a standardized method has not been established to detect the biofilm formation.
\end{abstract}

\section{Introduction}

Bloodstream infections (BSI) caused by various Candida spp. are a significant cause of morbidity and mortality especially in patients of intensive care units and patients with hematological malignancy. Although the introduction of antifungal drugs has improved the outcome of Candida BSIs, antifungal resistance has emerged recently as a major challenge in the management of
Candida infections because of the increasing prophylactic use of fluconazole and the relative rise in the proportion of non-albicans Candida species. ${ }^{1}$ Biofilm production on artificial or biological surfaces is an important virulence factor of Candida spp., because it is frequently associated with deep seated infections enhancing resistance to antimicrobial agents and protecting from host defenses, making these infections refractory to conventional therapy. ${ }^{2}$ Therefore, the detection of in vitro antifungal susceptibility and biofilm production of the agents is critical for the appropriate management of Candida bloodstream infection. In this study, primarily we aimed to investigate the biofilm formation rate and antifungal susceptibility characteristics for our bloodstream isolates. Secondarily, we aimed to evaluate two different biofilm detection methods.

\section{Materials and Methods}

\section{Isolates}

A total of 200 Candida species isolated from blood cultures were used; C. albicans $(\mathrm{n}=75), C$. parapsilosis $(\mathrm{n}=50)$, C. glabrata $(\mathrm{n}=30), C$. tropicalis $(\mathrm{n}=20), C . \quad$ krusei $(\mathrm{n}=10), C$. kefyr $(\mathrm{n}=4), C$. lusitaniae $(\mathrm{n}=4)$, C. dubliniensis $(\mathrm{n}=3), C$. guilliermondii $(\mathrm{n}=2)$ and $C$. pelliculosa $(\mathrm{n}=2)$. All isolates were selected randomly from the agents of bloodstream infection in our clinical microbiology laboratories from January 2008 to January 2014. Species identification was made by conventional procedures such as germ tube production, microscopic morphology on corn meal Tween 80 agar, as well as commercial methods such as CHROMagar Candida (BD Diagnostic, Sparks, MD), API 20C AUX (bioMérieux, Marcy L'Etoile, France). C. krusei ATCC 6258 and C. parapsilosis ATCC 22019 were used as quality control for antifungal susceptibility studies. All isolates were stored at $-70^{\circ} \mathrm{C}$ until use.

\section{Biofilm production}

The biofilms of Candida spp. were formed on presterilized, polystyrene, flat bottomed, 96-well microtiter plates (Nunclon; Nalge Nunc International, Roskilde, Denmark) as described previously. ${ }^{3-5}$ All isolates were grown for 24 $\mathrm{h}$ at $35^{\circ} \mathrm{C}$ on SDA, and saline washed suspensions of each isolate were prepared. The turbidity of each suspension was adjusted to the equivalent of $3 \times 10^{7} \mathrm{CFU} / \mathrm{mL}$ with Sabouraud dextrose broth (SDB) supplemented with glucose $(8 \%$ final concentra-
Correspondence: Yasemin Oz, Eskisehir Osmangazi University Medical Faculty, Department of Microbiology, Division of Mycology, Eskisehir, Turkey.

Tel.: +90.222.2392979-4550

E-mail: dryaseminoz@gmail.com

Key words: antifungal susceptibility, biofilm formation, bloodstream, Candida, XTT.

Contributions: YO designed the experiments; YO, IQ and EG performed the experimental work; YO evaluated the results and wrote the manuscript.

Conflict of interests: the authors declare no potential conflict of interests.

Received for publication: 3 October 2016

Revision received: 15 March 2017.

Accepted for publication: 30 March 2017.

This work is licensed under a Creative Commons Attribution NonCommercial 4.0 License (CC BY-NC 4.0).

(C) Copyright Y. Oz et al., 2017

Licensee PAGEPress, Italy

Microbiology Research 2017; 8:6916

doi:10.4081/mr.2017.6916

tion). Each well of microtiter plates was inoculated with aliquots of $20 \mu \mathrm{L}$ of yeast cell suspension and $180 \mu \mathrm{L}$ of SDB, three consecutive wells were used for each isolate. At least three wells included $200 \mu \mathrm{L}$ sterile SDB only as control wells in each plate. All plates were then incubated at $35^{\circ} \mathrm{C}$ without agitation. After $24 \mathrm{~h}$, all wells were discharged and washed once with distilled water (BIO-TEK EL $\times 50)$ to remove the planktonic cells; eventually $200 \mu \mathrm{L}$ of distilled water was added to each well. We used two different methods for detection of biofilm production; a spectrophotometric method and a colorimetric assay by using XTT [2,3-bis(2-methoxy-4-nitro-5-sulfophenyl)-2H-tetrazolium- 5-carboxanilide]. . $^{3-5}$

Firstly, biofilm was measured directly by spectrophotometric readings at $405 \mathrm{~nm}$ with a microtiter plate reader (BIO-TEK, EL $\times 800$, USA). The percent transmittance $(\% \mathrm{~T})$ was calculated by subtracting the $\% \mathrm{~T}$ value for each test sample from the $\% \mathrm{~T}$ value for the reagent blank to obtain a measure of the amount of light blocked passing through the wells (\%Tbloc). Biofilm production by each isolate was scored as negative $(\%$ Tbloc, $<5), 1+(\%$ Tbloc, 5 to 20$), 2+$ (\%Tbloc, 20 to 35), 3+ (\% Tbloc, 35 to 50$)$, or $4+(\%$ Tbloc, $>50))^{4,5}$ Each isolate was tested in duplicate.

In the second method, XTT (SigmaAldrich, St. Louis, MO, USA) was prepared 
in a saturated solution at $0.5 \mathrm{~g} / \mathrm{L}$ in Ringer's lactate and sterilized through a $0.22 \mu \mathrm{m}$ pore size filter. Menadione (Sigma-Aldrich, St. Louis, MO, USA) was prepared as 10 $\mathrm{mM}$ in acetone and added to XTT solution to be a $1 \mathrm{mM}$ final concentration. A $100-\mu 1$ aliquot of the XTT-menadione solution was then added to each prewashed biofilm and control wells (for the measurement of background XTT-reduction levels). The plates were then incubated at $37^{\circ} \mathrm{C}$ in the dark chamber for $2 \mathrm{~h}$. A colorimetric change in the XTT reduction assay, a direct correlation with the metabolic activity of the biofilm, was then measured in a microtiter plate reader at $490 \mathrm{~nm}^{3}$ The absorbance values of the controls were then subtracted from the values of the test wells to eliminate spurious results due to background interference. ${ }^{6}$ To compare the two different detection methods, net absorbance values of XTT reduction assay were classified as negative $(<0.2), 1+(0.2$ to 0.49$), 2+(0.5$ to 0.99$), 3+$ (1.0 to 1.49$)$, or $4+(1.5 \leq)$.

\section{Antifungal susceptibility testing}

Antifungal susceptibility testing was made by using reference broth microdilution (BMD) method, Clinical and Laboratory Standards Institute. ${ }^{7}$ First, caspofungin (CAS; Merck, Rahway, NJ, USA), anidulafungin (AND; Pfizer, New York, NY, USA) and amphotericin B (AmB; Sigma, St. Louis, MO, USA) were obtained as standard powders from their manufacturers. CAS was dissolved in distilled water; AND and AmB were dissolved in dimethyl sulfoxide. Stock solutions were prepared in RPMI 1640 medium (SigmaAldrich, Steinheim, Germany) buffered to pH 7.0 with 0.165 M morpholinepropanesulfonic acid (MOPS) buffer (SigmaAldrich, Steinheim, Germany), and serial twofold dilutions ranging $0.03-16 \mu \mathrm{g} / \mathrm{mL}$ of each drug were performed in 96 well microtiter plates.

All isolates were subcultured twice on Sabouraud dextrose agar (SDA) plates to ensure viability and purity. After 24- to 48$\mathrm{h}$ incubation, a standard $0.5 \mathrm{McF}$ arland fungal suspension was prepared with $0.85 \%$ sterile saline by spectrophotometric methods. It was diluted with RPMI 1640 broth medium to obtain a starting inoculum which results in $1-5 \times 10^{3}$ cells per $\mathrm{mL}$. Microtiter plates were inoculated and incubated at $35^{\circ} \mathrm{C}$. The minimum inhibitory concentrations (MICs) were read at $24 \mathrm{~h}$ for echinocandins, both 24 and $48 \mathrm{~h}$ for AmB. Endpoints for echinocandins were defined as the lowest concentration of drug that resulted in a prominent reduction (approximately $50 \%$ inhibition) of visual growth compared with the growth control wells. MICs of AmB were defined as the lowest concentration of drug which resulted in total inhibition of visual growth. For the analysis of antifungal susceptibility test results, interpretive species-specific MIC breakpoints were used for CAS and AND according to recently revised criteria by CLSI. ${ }^{8}$ All tests were carried out in duplicate.

\section{Statistical analysis}

Statistical analysis was performed using SPSS for Windows, version 20. All tests were two sided, and a $\mathrm{P}$ value of $<0.05$ was considered statistically significant.

\section{Results}

The results of biofilm production tests by both spectrophotometric and colorimetric XTT methods were presented in Table 1. Of all isolates, $41 \%$ and $55 \%$ produced apparent $(3+/ 4+)$ biofilm by spectrophotometric and XTT colorimetric methods, respectively. The most important biofilm production was observed among the isolates of C. tropicalis and C. parapsilosis by both detection methods. Although the biofilm formation in C. krusei isolates was very prominent, their number was low to generalize to this species in this study. In addition, C. glabrata had the lowest biofilm production with both methods. However, when we compared the two different detection methods, compliance was observed in $132(66 \%)$ isolates (Table 2); negative or low $(1+, 2+)$ biofilm production in 71 isolates and high $(3+, 4+)$ biofilm production in 61 isolates. When the biofilm formation was evaluated among these 132 isolates, significant species specific differences were observed $(\mathrm{P}=0.035)$; the highest biofilm production was seen for $C$. tropicalis isolates, followed by C. parapsilosis and C. glabrata was the lowest biofilm producer (Figure 1).

Antifungal susceptibility test results were summarized in Table 3. Although MICs of CAS and AND were high against the isolates of $C$. parapsilosis as expected, the lowest MIC values were observed with CAS and AND against other species. The highest AmB MICs were observed against C. krusei isolates.

\section{Discussion and Conclusions}

It is known that the incidence of nosocomial fungal infections has increased over the last few decades. Candidiasis, specifically candidemia, has been shown to be the most frequent mycotic infection of hospitalized patients and associated with a significant attributable mortality and excess length of hospital stay. ${ }^{9}$ Therefore, it is important to know the antifungal susceptibility characteristics for successfully management of these infections. Although C. albicans is the most frequently isolated species, recent reports indicate a trend toward an increasing prevalence of infections caused by species of non-albicans Candida such as $C$. parapsilosis, C. tropicalis, and $C$. glabrata. ${ }^{9,10}$ In our study, we evaluated the biofilm production rate and the susceptibility characteristics for three antifungals of our bloodstream isolates. For this reason, we selected the species reflecting our bloodstream Candida isolate collection and

Table 1. The results of methods used for the detection of biofilm production.

\begin{tabular}{|c|c|c|c|c|c|c|c|c|c|c|}
\hline \multirow[t]{3}{*}{ Species } & \multicolumn{10}{|c|}{ Biofilm production } \\
\hline & \multicolumn{5}{|c|}{ With spectrophotometric method (\%) } & \multicolumn{5}{|c|}{ With XTT colorimetric method (\%) } \\
\hline & Neg & + & ++ & +++ & ++++ & Neg & + & ++ & +++ & ++++ \\
\hline C. albicans & $15(20)$ & $16(21)$ & $14(19)$ & $9(12)$ & $21(28)$ & $1(1)$ & $16(22)$ & $22(29)$ & $22(29)$ & $14(19)$ \\
\hline C. parapsilosis & $1(2)$ & $6(12)$ & $20(40)$ & $12(24)$ & $11(22)$ & 0 & $3(6)$ & $15(30)$ & $25(50)$ & $7(14)$ \\
\hline C. glabrata & $11(37)$ & $5(17)$ & $8(27)$ & $2(6)$ & $4(13)$ & $5(17)$ & $4(13)$ & $9(30)$ & $11(37)$ & $1(3)$ \\
\hline C. tropicalis & $4(20)$ & $2(10)$ & $4(20)$ & $4(20)$ & $6(30)$ & 0 & $3(15)$ & $3(15)$ & $6(30)$ & $8(40)$ \\
\hline C. krusei & 0 & 1 & 1 & 8 & 0 & 0 & 1 & 2 & 4 & 3 \\
\hline Candida spp. & 6 & 2 & 2 & 1 & 4 & 0 & 1 & 6 & 6 & 2 \\
\hline Total & 37 (18.5) & $32(16)$ & 49 (24.5) & $36(18)$ & $46(23)$ & $6(3)$ & $28(14)$ & 57 (28.5) & $74(37)$ & $35(17.5)$ \\
\hline
\end{tabular}


three antifungal drugs in the treatment of invasive Candida infections.

Currently, there is no standardized method for the detection of biofilm formation; therefore, we used two different well established methods, spectrophotometric and colorimetric XTT. We observed significant species specific differences in terms of biofilm production; while the strongest biofilm producer species was $C$. tropicalis, the weakest one was $C$. glabrata by both methods. Tumbarello et al..$^{5}$ reported that biofilm production was most frequently observed for isolates of $C$. tropicalis (71.4\%), followed by C. glabrata (23.1\%), C. albicans $(22.6 \%)$, C. parapsilosis $(21.8 \%)$; the highest relative intensity of biofilm formation was seen for $C$. tropicalis isolates, followed by $C$. parapsilosis among biofilm-positive isolates. Both the highest frequency and the highest relative intensity of biofilm formation were seen for C. tropicalis isolates, followed by C. parapsilosis in our study (Table 2). Pannanusorn et al. ${ }^{11}$ evaluated the biofilm formation of 393 bloodstream isolates by XTT colorimetric method with some modifications; they reported a high biofilm production rate $(58.8 \%)$ for all isolates and $88.7 \%$ for nonalbicans Candida species. In addition, while all of C. tropicalis and C. krusei isolates, $95 \%$ of $C$. glabrata isolates, $66.7 \%$ of C. parapsilosis isolates, $40.3 \%$ of C. albi-

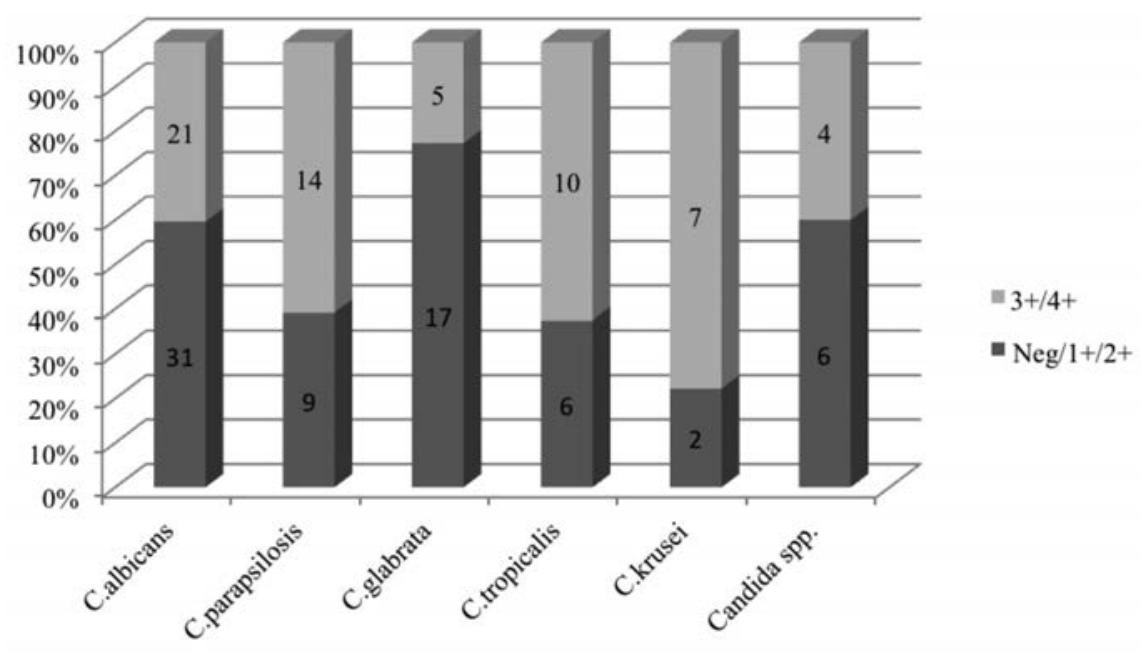

Figure 1. The distribution of biofilm production rate among Candida species. This Figure was constituted by using the compatible results of two different biofilm detection methods (italicized in Table 2).

Table 2. The comparison of the biofilm detection methods.

\begin{tabular}{|c|c|c|c|c|c|}
\hline \multirow{2}{*}{ Spectrophotometric } & \multicolumn{5}{|c|}{ XTT colorimetric } \\
\hline & Negative & + & ++ & +++ & ++++ \\
\hline Negative & 3 & 8 & 24 & 0 & 0 \\
\hline+ & 1 & 5 & 9 & 14 & 0 \\
\hline++ & 2 & 9 & 10 & 19 & 9 \\
\hline+++ & 8 & 8 & 11 & 13 & 8 \\
\hline++++ & 0 & 0 & 7 & 22 & 18 \\
\hline
\end{tabular}

The compatible results between the two detection methods are italicized.

Table 3 Antifungal susceptibility test results by broth microdilution methods.

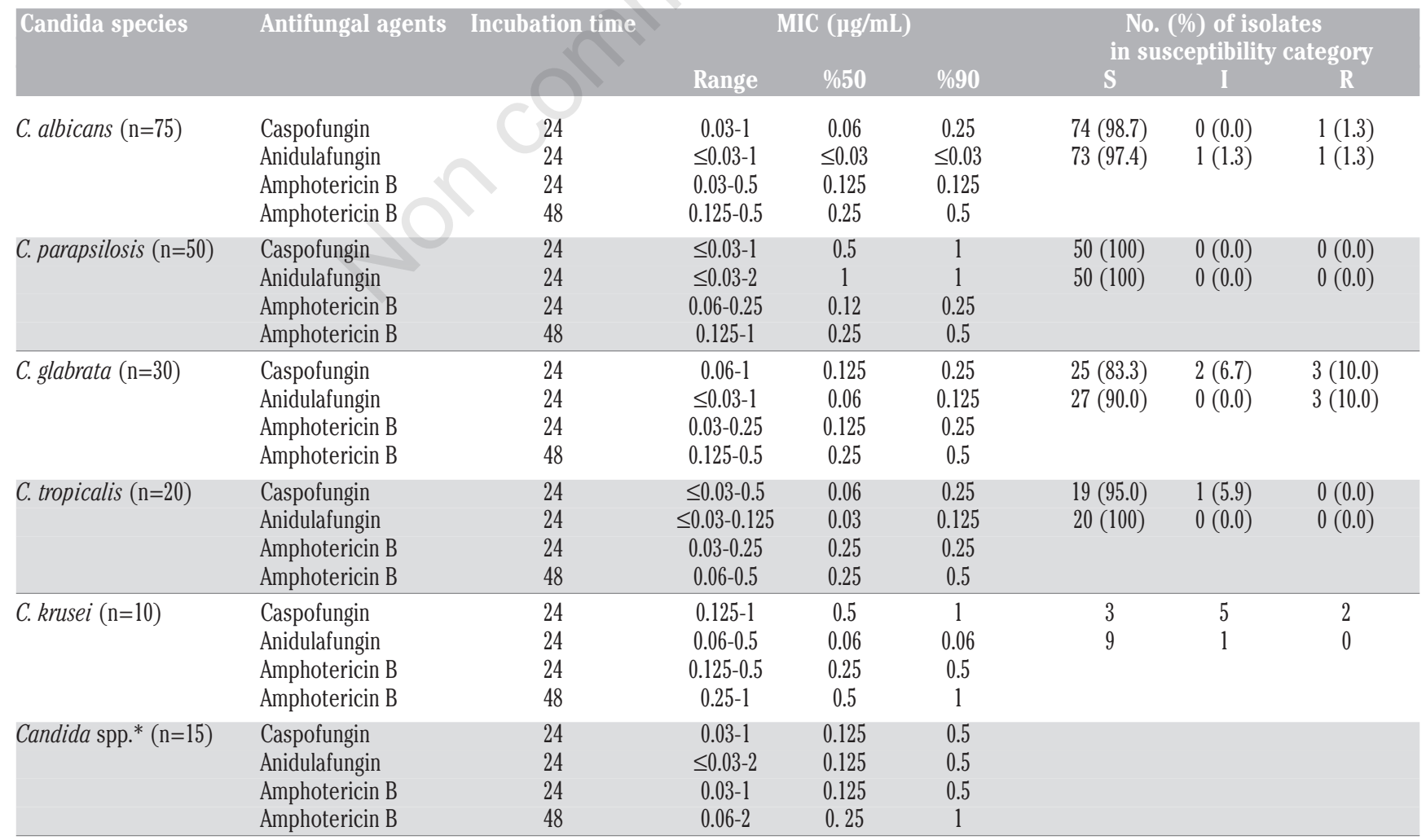

${ }^{*}$ C. kefyr, C. lusitaniae , C. guilliermondii, C. pelliculosa and other Candida spp. I, intermediate; R, resistant; S susceptible. 
cans isolates were found biofilm positive, high biofilm production capacity was observed in the rates of $87 \%$ in C. tropicalis, $69 \%$ in C. glabrata, $45.5 \%$ in C. parapsilosis, and $11 \%$ in $C$. albicans in their study. ${ }^{11}$ Although the rates of biofilm production showed some variations depending on the detection method used, it was species specific and the most frequent biofilm producer species was $C$. tropicalis. The consistency between biofilm detection methods was found low in our study. In another study using the same biofilm detection methods, authors reported that the results of spectrophotometric method correlated well with XTT absorbance measurements for all isolates tested. ${ }^{5}$ Dhale et al. ${ }^{12}$ compared three different methods, spectrophotometric and colorimetric methods, and crystal violet assay; they did not find significant difference in proportion of biofilm positive cases detected by the three methods. However, Kuhn et al. ${ }^{13}$ evaluated the species- and strain-related tetrazolium metabolism in $C$. albicans and $C$. parapsilosis by using XTT. They reported that the use of colorimetric XTT method for the comparison of different Candida isolates may have some limitations, for instance different strains metabolize XTT with different capabilities, a linear relationship may not appear between organism number and colorimetric signal, and the relationship between the XTT concentration and the resultant colorimetric signal is not necessarily proportional. ${ }^{13}$ Although XTT reduction assay is seen as a convenient method for investigating the behavior of yeast, it may not always be appropriate. ${ }^{13}$ We think that the use of more than one test for biofilm detection may be useful because it seems that the detection of biofilm formation is still problematic by using these tests.

A remarkable resistance was not observed among our isolates against all antifungals in our study. We observed the resistance to echinocandin in a low proportion among the isolates of $C$. albicans, $C$. glabrata and $C$. krusei. In a study evaluating the antifungal susceptibility of 1214 bloodstream yeast isolates by using a commercial colorimetric microdilution plate, the susceptibility rates of echinocandins were very high, resistance was reported only among the isolates of $C$. albicans ( $0.2 \%$ for both CAS and AND) and C. krusei $\left(9.1 \%\right.$ for CAS only). ${ }^{14}$ Pfaller et al. ${ }^{15,16}$ have reported that both species distribution and antifungal resistance patterns vary across geographic regions; resistance to caspofungin (0-1.6\%) and anidulafungin (0$2.4 \%$ ) was low and most prevalent among C. glabrata isolates. Tan et al. ${ }^{17}$ evaluated the antifungal susceptibilities of 861 invasive bloodstream isolates of Candida species in the Asia-Pacific region, they reported that the resistance to echinocandins remained very low, reduced susceptibility or resistance was the most prominent for CAS against C. glabrata isolates. The MIC breakpoint values for AmB were not established yet; however, the most of our isolates had low MIC values for this antifungal. As a result, biofilm formation is an important virulence factor of Candida species, and the rate of biofilm production among our bloodstream isolates was found considerably high. The most important biofilm production was observed among the isolates of C. tropicalis and C. parapsilosis; C. glabrata had the lowest biofilm production rate. A certain standardized method has not been established to detect the biofilm formation for yeasts, and the consistency rate between biofilm detection methods was found $66 \%$ in this study. Therefore we think that further studies are necessary for the standardization of detection the biofilm formation in Candida spp.

\section{References}

1. Dewan E, Biswas D, Kakati B, et al. Epidemiological and mycological characteristics of candidemia in patients with hematological malignancies attending a tertiary-care center in India. Hematol Oncol Stem Cell Ther 2015;8:99-105.

2. Ramage G, Saville SP, Thomas DP, López-Ribot JL. Candida biofilms: an update. Eukaryot Cell 2005;4:633-8.

3. Ramage G, Vande Walle K, Wickes BL, López-Ribot JL. Standardized method for in vitro antifungal susceptibility testing of Candida albicans biofilms. Antimicrob Agents Chemother 2001;45: 2475-9.

4. Shin JH, Kee SJ, Shin MG, et al. Biofilm production by isolates of Candida species recovered from nonneutropenic patients: comparison of bloodstream isolates with isolates from other sources. J Clin Microbiol 2002; 40:1244-8.

5. Tumbarello M, Posteraro B, Trecarichi EM, et al. Biofilm production by Candida species and inadequate antifungal therapy as predictors of mortality for patients with candidemia. J Clin Microbiol 2007;45:1843-50.

6. Hiller E, Heine S, Brunner H, Rupp S. Candida albicans Sun41p, a putative glycosidase, is involved in morphogenesis, cell wall biogenesis, and biofilm formation. Eukaryot Cell 2007;6:2056-65.

7. CLSI. Reference Method for Broth Dilution Antifungal Susceptibility Testing of Yeasts; Approved Standard Third Edition. CLSI document M27-A3.
Wayne, PA: Clinical and Laboratory Standards Institute. 2008

8. CLSI. Reference Method for Broth Dilution Antifungal Susceptibility Testing of Yeasts; Fourth Informational Supplement. CLSI document M27-S4. Wayne, PA: Clinical and Laboratory Standards Institute. 2012.

9. Pfaller MA, Diekema DJ. Role of sentinel surveillance of candidemia: trends in species distribution and antifungal susceptibility. J Clin Microbiol 2002;40: 3551-7.

10. Tortorano AM, Peman J, Bernhardt H, et al. Epidemiology of candidaemia in Europe: results of 28-month European Confederation of Medical Mycology (ECMM) hospital-based surveillance study. Eur J Clin Microbiol Infect Dis 2004;23:317-22.

11. Pannanusorn S, Fernandez V, Römling U. Prevalence of biofilm formation in clinical isolates of Candida species causing bloodstream infection. Mycoses 2013;56:264-72.

12. Dhale RP, Ghorpade MV, Dharmadhikari CA. Comparison of various methods used to detect biofilm production of Candida species. J Clin Diagn Res 2014;8:DC18-c20.

13. Kuhn DM, Balkis M, Chandra J, et al. Uses and limitations of the XTT assay in studies of Candida growth and metabolism. J Clin Microbiol 2003;41:506-8.

14. Posteraro B, Spanu T, Fiori B, et al. Antifungal susceptibility profiles of bloodstream yeast isolates by Sensititre YeastOne over nine years at a large Italian teaching hospital. Antimicrob Agents Chemother 2015;59:3944-55.

15. Pfaller MA, Moet GJ, Messer SA, et al. Geographic variations in species distribution and echinocandin and azole antifungal resistance rates among Candida bloodstream infection isolates: report from the SENTRY Antimicrobial Surveillance Program (2008 to 2009). J Clin Microbiol 2011;49:396-9.

16. Pfaller MA, Messer SA, Woosley LN, et al. Echinocandin and triazole antifungal susceptibility profiles for clinical opportunistic yeast and mold isolates collected from 2010 to 2011: application of new CLSI clinical breakpoints and epidemiological cutoff values for characterization of geographic and temporal trends of antifungal resistance. J Clin Microbiol 2013;51:2571-81.

17. Tan TY, Hsu LY, Alejandria MM, et al. Antifungal susceptibility of invasive Candida bloodstream isolates from the Asia-Pacific region. Med Mycol 2016;54:471-7. 